\title{
Investigation of Unsteady Mixed Convection Flow near the Stagnation Point of a Heated Vertical Plate embedded in a Nanofluid-Saturated Porous Medium by Self-Similar Technique
}

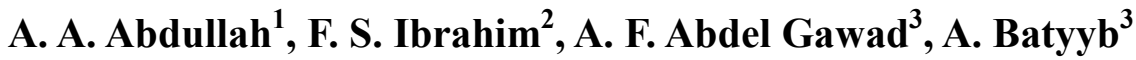 \\ ${ }^{1}$ Department of Mathematical Sciences, Umm Al-Qura University, Makkah, Saudi Arabia \\ ${ }^{2}$ Department of Mathematics, University College, Umm Al-Qura University, Makkah, Saudi Arabia \\ ${ }^{3}$ Mech. Eng. Dept., College of Engineering and Islamic Architecture, Umm Al-Qura University, Makkah, Saudi Arabia
}

Email address:

batyyb@hotmail.com (A. Batyyb)

To cite this article:

A. A. Abdullah, F. S. Ibrahim, A. F. Abdel Gawad, A. Batyyb. Investigation of Unsteady Mixed Convection Flow near the Stagnation Point of a Heated Vertical Plate embedded in a Nanofluid-Saturated Porous Medium by Self-Similar Technique. American Journal of Energy Engineering. Special Issue: Computational Simulation of Fire and Evacuation. Vol. 3, No. 4-1, 2015, pp. 42-51.

doi: 10.11648/j.ajee.s.2015030401.13

\begin{abstract}
This paper aims to study the problem of unsteady mixed convection in a stagnation flow on a heated vertical surface embedded in a nanofluid-saturated porous medium. The employed mathematical model for the nanofluid takes into account the effects of Brownian motion and thermophoresis. The presence of a solid matrix, which exerts first and second resistance parameters, is considered in this study. The self-similar solutions for the system of equations governing the problem are obtained. The resulting system of ordinary differential equations that govern the flow is solved numerically using fourthfifth order Runge-Kutta with shooting method. Numerical results for the dimensionless velocity, temperature and nanoparticle volume fraction as well as skin friction, Nusselt number and Sherwood number are produced for different values of the influence parameters.
\end{abstract}

Keywords: Unsteady Mixed Convection, Self-Similar Solution, Nanofluids, Stagnation, Porous Media

\section{Introduction}

The study of mixed convection flow has applications in several industrial and technical processes such as nuclear reactors cooled during emergency shutdown, solar centralreceivers exposed to winds, electronic devices cooling by fans, heat exchanges placed in a low-velocity environment, etc. The mixed convection flows become important when the buoyancy forces, due to the temperature difference between the wall and the free stream, become large. The mixed convection flow in the stagnation flow region of a vertical plate has been investigated by Ramachandra et al. [1].

When there is an impulsive change in the velocity field, the inviscid flow develops instantaneously, but the flow in the viscous layer near the wall develops slowly which becomes fully-developed steady flow after sometime. For a small period of time, the flow is dominated by the viscous forces and the unsteady acceleration, but for a large period of time, it is dominated by the viscous forces, the pressure gradient and the convective acceleration. Scshadri et al. [2] studied the unsteady mixed convection flow in the stagnation region of a heated vertical plate due to impulsive motion. The boundary-layer flow development of a viscous fluid on a semi-infinite flat plate due to impulsive motion of the free stream was investigated by Hall [3], Dennis [4] and Watkins [5]. The corresponding problem over a wedge was studied by Smith [6], Nanbu [7], and Williams and Rhyne [8].

Kumari [9] examined the temporal development of momentum and thermal boundary layers on an impulsivelystarted wedge with a magnetic field and has obtained the solution numerically starting from the initial steady state to the final steady state. The flow development of the laminar boundary on an impulsively-started translating and spinning rotational symmetric body was considered by Ece [10]. Qzturk and Ece [11] studied the unsteady forced convection heat transfer from a translating and spinning body. Brown and Riley [12] presented an analysis that covered three 
distinct phases in the temporal development of the free convection flow past a suddenly heated semi-infinite vertical plate. The unsteadiness in the flow field arises due to the step change in wall temperature. Ingham [13] has considered essentially the same problem as that of Brown and Riley [12] but instead of taking the step change in wall temperature, the wall temperature $T_{\infty}$ is suddenly raised to $T_{w}=T_{\infty}+A x^{m}$, where $A$ is a positive constant, $m$ is a constant and $x$ is the distance measured from the leading edge of the plate.

The mixed convection flow at a two-dimensional stagnation point was investigated by Amin and Riley [14]. The forced flow is a stagnation point flow and the free convection part is due to a pressure gradient that is induced by temperature variations along the boundary. Hassanien, et al. [15] analyzed the problem of unsteady free convection flow in the stagnation-point region of a rotating sphere embedded in a porous medium. The unsteady flow and heat transfer of a viscous fluid in the stagnation region of a threedimensional body embedded in a porous medium was investigated by Hassanien, et al. [16]. Hassanien and AlArab [17] studied the problem of thermal radiation and variable viscosity effects on unsteady mixed convection flow in the stagnation region on a vertical surface embedded in a porous medium with surface heat flux

The research topic of nanofluids has received considerable interest worldwide. Inherently low thermal conductivity is a primary limitation in developing energy-efficient heat transfer fluids that are required for ultrahigh-performance cooling. A very small amount of guest nanoparticles, when dispersed uniformly and suspended stably in host fluids, can provide dramatic improvements in the thermal properties of the host fluids. According to Yacob et al. [18], nanofluids are produced by dispersing the nanometer-scale solid particles into base liquids with low thermal conductivity such as water and ethylene glycol. Nanoparticles are usually made of metal, metal oxide, carbide, nitride and even immiscible nano-scale liquid droplets. Congedo et al. [19] compared different models of nanofluid (regarded as a single phase) to investigate the density, specific heat, viscosity and thermal conductivity and discussed the water- $\mathrm{A} 12 \mathrm{O} 3$ nanofluid in details by using CFD. Hamad et al. [20] introduced a oneparameter group to represent similarity reductions for the problem of magnetic field effects on free-convective nanofluid flow past a semi-infinite vertical flat plate following a nanofluid model proposed by Buongiorno [21]. Hamad [22] obtained the analytical solutions for convective flow and heat transfer of a viscous incompressible nanofluid past a semi-infinite vertical stretching sheet in the presence of magnetic field. Khan and Pop [23] obtained similarity solutions depending on Prandtl, Lewis, Brownian motion and thermophoresis numbers on the steady boundary-layer flow, heat and mass over a stretching surface in its plane. Further, Abu-Nada and Chamkha [24] presented the natural convection heat transfer characteristics in a differentiallyheated enclosure filled with a $\mathrm{CuO}-\mathrm{EG}-$ water nanofluid for different variable thermal conductivity and variable viscosity models. For more information, see also Das et al. [25], and
Kakaç and Pramuanjaroenkij [26]. Muthtamilselvan et al. [27] claimed that it is difficult to have a precise idea on how nanoparticles enhance the heat transfer characteristics of nanofluids.

In this study, our main objective is to analyze mixed convection in stagnation flow on a heated vertical surface embedded in a nanofluid-saturated porous medium. The effect of the presence of an isotropic solid matrix due to impulsive motion is considered. Moreover, we examine the combined effect of Brownian motion, thermophoresis parameters and nanoparticle fraction on boundary-layer flow and heat transfer and due to nanofluid. The governing boundary layer equations are transformed to a two-point boundary-value problem using similarity variables. These are numerically solved using fourth-fifth order Runge-Kutta method with shooting technique. The effects of governing parameters on fluid velocity, temperature and particle concentration are discussed and shown graphically and in tables as well.

\section{Mathematical Analysis}

Let us consider a semi-infinite vertical plate embedded in a saturated porous medium with temperature $T_{w}$ and concentration $\phi_{w}$. The ambient temperature and concentration, respectively are $T_{\infty}$ and. $\phi_{\infty}$. At $t=0.0$ the ambient fluid is impulsively moved with a velocity $U_{e}$ and at the same time the surface temperature is suddenly raised. The flow field is over a heated vertical surface where the upper half of the field is assisted by the buoyancy force. However, the buoyancy force opposes the lower part. The surface of the plate is assumed to have arbitrary temperature and concentration. The physical flow model and coordinate system is shown in Fig. 1.

Under above assumptions along with Boussinesq and boundary layer approximations, the governing equations of the conservation of mass, momentum, energy and nanoparticles volume fraction can be expressed as:

$$
\begin{gathered}
\frac{\partial u}{\partial x}+\frac{\partial v}{\partial y}=0 \\
\frac{\partial u}{\partial t}+u \frac{\partial u}{\partial x}+v \frac{\partial u}{\partial y}=\frac{\partial U_{e}}{\partial t}+U_{e} \frac{\partial U_{e}}{\partial x}+v \frac{\partial^{2} u}{\partial y^{2}}+\frac{v}{K}\left(U_{e}-u\right) \\
+\frac{\Gamma}{K^{1 / 2}}\left(U_{e}^{2}-u^{2}\right)+\left[\left(1-\phi_{\infty}\right) \beta\left(T-T_{\infty}\right)\right. \\
\left.-\left(\rho_{p}-\rho_{f}\right)\left(\phi-\phi_{\infty}\right) / \rho_{f}\right] g \\
\frac{\partial T}{\partial t}+u \frac{\partial T}{\partial x}+v \frac{\partial T}{\partial y}=\alpha \frac{\partial^{2} T}{\partial y^{2}}+\tau\left[D_{B} \frac{\partial T}{\partial y} \frac{\partial \phi}{\partial y}+\frac{D_{T}}{T_{\infty}}\left(\frac{\partial T}{\partial y}\right)^{2}\right] \\
\frac{\partial \phi}{\partial t}+\left(u \frac{\partial \varphi}{\partial x}+v \frac{\partial \phi}{\partial y}\right)=D_{B} \frac{\partial^{2} \phi}{\partial y^{2}}+\frac{D_{T}}{T_{\infty}}\left(\frac{\partial^{2} T}{\partial y^{2}}\right)^{\prime}
\end{gathered}
$$

Where, $x$ and $y$ are the coordinates along and normal to the 
surface, respectively. $v$ is the kinematic viscosity, time is denoted by $t, u$ and $v$ are the velocity components along the $x$ and $y$ directions, respectively. $K$ is the permeability of the porous medium and $\Gamma$ is the empirical constant in the second-order resistance. $T$ is the temperature, $\phi$ is the solid volume fraction, $\alpha=k /(\rho C)_{f}$ is the thermal diffusivity, $D_{B}$

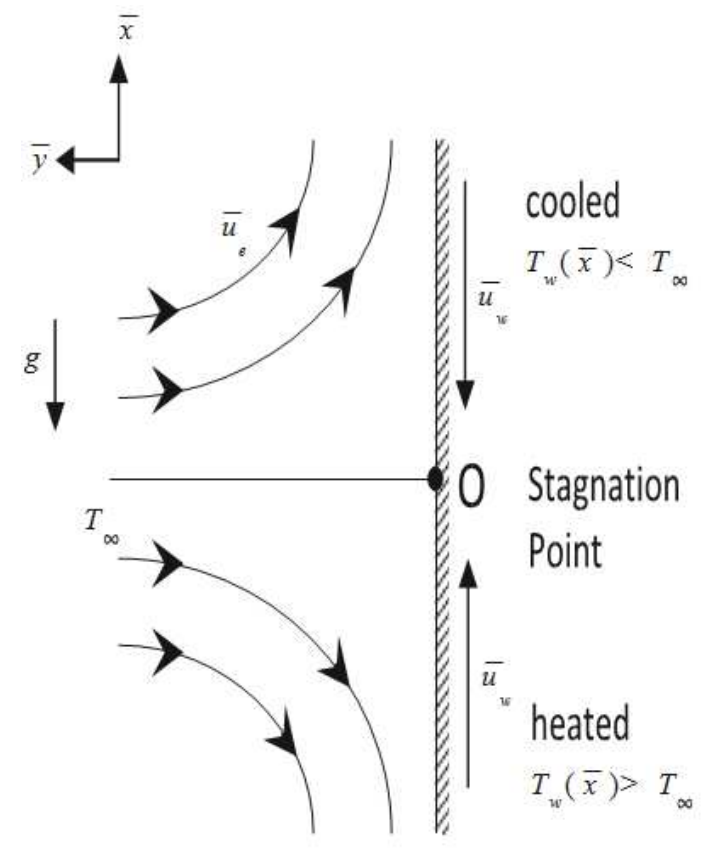

(a)Assisting Flow is the Brownian diffusion coefficient, $D_{T}$ is the thermophoretic diffusion coefficient, $\tau=(\rho C)_{p} /(\rho C)_{f}$ is the ratio of the effective heat capacity of the nanoparticle material to the heat capacity of the fluid, $g$ is the acceleration due to gravity.

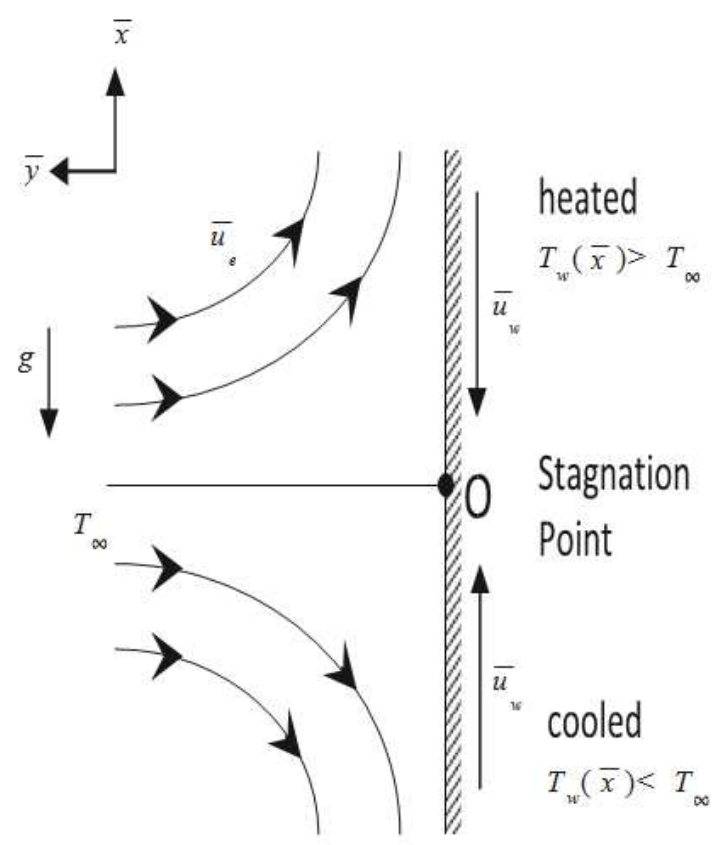

Fig. 1. Physical model and coordinate system.

The proposed initial conditions are

$$
\begin{aligned}
& u(x, y, t)=v(x, y, t)=0, \quad T(x, y, t)=T_{\infty}, \quad \phi(x, y, t)=\phi_{\infty} \text { for } \\
& t<0 \text {. }
\end{aligned}
$$

The proposed boundary conditions for $t \geq 0$ are

$$
\begin{aligned}
& u(t, x, 0)=v(t, x, 0)=0, \quad u(t, x, \infty)=U_{e}=a x /(1-c t), \quad a>0, \\
& T(t, x, 0)=T_{w}(t, x)=T_{\infty}+b x /(1-c t)^{2}, \quad T(t, x, \infty)=T_{\infty}, \\
& \phi(t, x, 0)=\phi_{w}(t, x)=\phi_{\infty}+b x /(1-c t)^{2}, \phi(t, x, \infty)=\phi_{\infty}, b, c>0,
\end{aligned}
$$

where a and $\mathrm{c}$ are constants (with $\mathrm{a}>0$ and $\mathrm{c} \geq 0$, where ct $<$ 1 ), and both have dimension time ${ }^{-1}$, while $b$ is a constant and has dimension temperature/length, with $\mathrm{b}>0$ and $\mathrm{b}<0$ corresponding to the assisting and opposing flows, respectively, and $b=0$ is for forced convection limit (absence of buoyancy force).

To transform equations (1-4) into a set of ordinary differential equations, we now introduce the following dimensionless quantities; the mathematical analysis of the problem is simplified by introducing the following similarity transforms:

$$
\begin{aligned}
& \eta=\sqrt{\frac{a}{v(1-c t)}} y, \quad \psi=\sqrt{\frac{a v}{1-c t}} x f(\eta), \\
& \theta(\eta)=\frac{T-T_{\infty}}{T_{w}-T_{\infty}}, \quad \varphi(\eta)=\frac{\phi-\phi_{\infty}}{\phi_{w}-\phi_{\infty}}
\end{aligned}
$$

The equation of continuity is satisfied if we choose a stream function $\psi(x, y)$ such that $u=\frac{\partial \psi}{\partial y}, v=-\frac{\partial \psi}{\partial x}$.

Using the similarity transformation quantities (7), the governing Eqs. (2-6) are transformed to the ordinary differential equation as follows:

$$
\begin{aligned}
& f^{\prime \prime \prime}+1+A\left(1-f^{\prime}+\frac{1}{2} \eta f^{\prime \prime}\right)-f^{\prime 2}+f f^{\prime \prime}+\lambda\left(\theta-N_{r} \varphi\right) \\
& +\gamma\left(1-f^{\prime}\right)+\Delta\left(1-f^{\prime 2}\right)=0 \\
& \frac{1}{\operatorname{Pr}} \theta^{\prime \prime}-A\left(2 \theta+\frac{1}{2} \eta \theta^{\prime}\right)-f^{\prime} \theta+f \theta^{\prime}+N_{b} \theta^{\prime} \varphi^{\prime}+N_{t} \theta^{2}=0 \\
& \frac{1}{L e} \varphi^{\prime \prime}-A\left(2 \varphi+\frac{1}{2} \eta \varphi^{\prime}\right)-f^{\prime} \varphi+f \varphi^{\prime}+\frac{1}{L e} \frac{N_{t}}{N_{b}} \theta^{\prime \prime}=0
\end{aligned}
$$

With the boundary conditions: 


$$
\begin{aligned}
& \mathrm{f}^{\prime}(\eta)=0, \quad \mathrm{f}(\eta)=0, \theta(\eta)=0, \quad \varphi(\eta)=0, \\
& \text { for } t<0,
\end{aligned}
$$

and proposed boundary conditions for $t \geq 0$ are

$$
\begin{aligned}
& \mathrm{f}(0)=0, \quad \mathrm{f}^{\prime}(0)=0, \quad \theta(0)=1, \quad \varphi(0)=1, \\
& \mathrm{f}^{\prime}(\infty)=1, \theta(\infty)=0, \quad \varphi(\infty)=0,
\end{aligned}
$$

Where the governing parameters are defined as:

$$
\begin{aligned}
& A=\frac{c}{a}, \quad \operatorname{Pr}=\frac{v}{\alpha}, \quad L e=\frac{v}{D_{B}}, \lambda=\frac{G r_{x}}{\operatorname{Re}_{x}^{2}}, \\
& \gamma=\frac{1}{D a_{x} \operatorname{Re}_{x}}, \Delta=\frac{\Gamma}{D a_{x}^{1 / 2}} N_{b}=\frac{\tau}{v} D_{B}\left(\phi_{w}-\phi_{\infty}\right), \\
& N_{t}=\frac{\tau}{v}\left(\frac{D_{T}}{T_{\infty}}\right)\left(T_{w}-T_{\infty}\right), N_{r}=\frac{\left(\rho_{p}-\rho_{f}\right)\left(\phi_{w}-\phi_{\infty}\right)}{\rho_{f} \beta_{T}\left(1-\phi_{\infty}\right)\left(T_{w}-T_{\infty}\right)}
\end{aligned}
$$

Where $f^{\prime}, \theta$ and $\varphi$ are the dimensionless velocity, temperature and particle concentration, respectively. The prime denotes differentiation with respect to the similarity variable $\eta . G r_{x}=\frac{g \beta\left(T_{w}-T_{\infty}\right) x^{3}}{v^{2}}, \operatorname{Re}_{x}=\frac{U_{e} x}{v} \quad$ and $D a_{x}=\frac{K}{x^{2}}$ are Grashof, Reynolds and Darcy numbers, respectively. $A, \lambda, P r, L e, N_{T}, N_{B}, N_{r}, \gamma$ and $\Delta$ denotes the unsteadiness parameter, mixed convection parameter, Prandtl number, Lewis number, thermophoresis parameter, Brownian motion parameter, nanofluid buoyancy ratio, first resistant parameter and second resistant parameter, respectively.

The important physical quantities of interest in this problem are the skin friction coefficient (wall shear stress) $C_{f}$, local Nusselt number $N u_{x}$ and the local Sherwood number $S h_{x}$ that are defined as:

$$
\begin{aligned}
& C_{f}=\frac{\mu}{\rho U_{\infty}^{2}}\left(\frac{\partial u}{\partial y}\right)_{y=0}, \quad N u_{x}=\frac{x}{\left(T_{w}-T_{\infty}\right)}\left(\frac{\partial \theta}{\partial y}\right)_{y=0}, \\
& S h_{x}=\frac{x}{\left(\phi_{w}-\phi_{\infty}\right)}\left(\frac{\partial \phi}{\partial y}\right)_{y=0}
\end{aligned}
$$

Substituting Eq. (7) into Eq. (18), we get

$$
\begin{aligned}
& C_{f}=\operatorname{Re}_{x}^{-1 / 2} f^{\prime \prime}(0), \quad N u_{x}=-\operatorname{Re}_{x}^{-1 / 2} \theta^{\prime}(0), \\
& S h_{x}=-\operatorname{Re}_{x}^{-1 / 2} \varphi^{\prime}(0)
\end{aligned}
$$

\section{Results and Discussion}

The resulting differential systems (8)-(10) subjected to the boundary conditions (12) are solved numerically through fourth-fifth order Runge-Kutta method (RK45) using a shooting technique. The values of the governing parameters are chosen arbitrary. However, the numerical results are presented for some representative values of these governing parameters. In order to see the physical insight, the numerical values of velocity $f^{\prime}(\eta)$, temperature $\theta(\eta)$, and nanoparticle volume fraction $\varphi(\eta)$ with the boundary layer have been computed for different parameters as unsteadiness parameter $A$, mixed convection parameter $\lambda$, nanofluid buoyancy ratio parameter $N_{r}$, thermophoresis parameter $N_{t}$, Brownian motion parameter $N_{b}$, first resistant parameter $\gamma$, second resistant parameter $\Delta$. Prandtl number Pr and Lewis number $L e$.

\begin{tabular}{|c|c|c|c|c|c|c|c|c|c|c|}
\hline \multirow{2}{*}{$\mathbf{N}_{\mathrm{b}}$} & \multirow{2}{*}{$\mathbf{N}_{\mathrm{t}}$} & \multicolumn{3}{|l|}{$\mathrm{N}_{\mathrm{r}}=0.5$} & \multicolumn{3}{|l|}{$N_{r}=1$} & \multicolumn{3}{|l|}{$N_{r}=3$} \\
\hline & & $f^{f^{\prime}}(0)$ & $-\theta^{\prime}(0)$ & $-\phi^{\prime}(0)$ & $f^{\prime}(0)$ & $-\theta^{\prime}(0)$ & $-\phi^{\prime}(0)$ & $f^{\prime \prime}(0)$ & $-\theta^{\prime}(0)$ & $-\phi^{\prime}(0)$ \\
\hline \multirow{5}{*}{0.1} & 0.1 & 1.86358 & 1.98266 & 2.99959 & 1.75281 & 1.97640 & 2.97942 & 1.29405 & 1.94971 & 2.89209 \\
\hline & 0.3 & 1.87689 & 1.46341 & 3.00885 & 1.74724 & 1.46030 & 2.96609 & 1.20280 & 1.44720 & 2.77490 \\
\hline & 0.5 & 1.89459 & 1.18916 & 3.30288 & 1.75777 & 1.18808 & 3.23693 & 1.17626 & 1.18396 & 2.93645 \\
\hline & 0.7 & 1.91206 & 1.02290 & 3.60614 & 1.77309 & 1.02289 & 3.52129 & 1.17597 & 1.02359 & 3.12943 \\
\hline & 0.9 & 1.92805 & 0.91043 & 3.88186 & 1.78917 & 0.91098 & 3.78222 & 1.18647 & 0.91421 & 3.31690 \\
\hline \multirow{5}{*}{0.3} & 0.1 & 1.91563 & 1.10471 & 3.50357 & 1.82008 & 1.10377 & 3.48831 & 1.42727 & 1.09997 & 3.42351 \\
\hline & 0.3 & 1.93714 & 0.89987 & 3.66024 & 1.83864 & 0.89972 & 3.64082 & 1.43308 & 0.89932 & 3.55799 \\
\hline & 0.5 & 1.95564 & 0.78110 & 3.80811 & 1.85644 & 0.78133 & 3.78544 & 1.44766 & 0.78250 & 3.68860 \\
\hline & 0.7 & 1.97137 & 0.70268 & 3.93471 & 1.87243 & 0.70308 & 3.90958 & 1.46449 & 0.70499 & 3.80212 \\
\hline & 0.9 & 1.98482 & 0.64593 & 4.04428 & 1.88657 & 0.64642 & 4.01722 & 1.48123 & 0.64867 & 3.90151 \\
\hline \multirow{5}{*}{0.5} & 0.1 & 1.94644 & 0.69021 & 3.53118 & 1.85358 & 0.69053 & 3.51722 & 1.47246 & 0.69207 & 3.45816 \\
\hline & 0.3 & 1.96535 & 0.60599 & 3.66488 & 1.87203 & 0.60649 & 3.64911 & 1.48897 & 0.60871 & 3.58235 \\
\hline & 0.5 & 1.98126 & 0.55157 & 3.77327 & 1.88824 & 0.55213 & 3.75618 & 1.50642 & 0.55460 & 3.68382 \\
\hline & 0.7 & 1.99473 & 0.51253 & 3.86302 & 1.90230 & 0.51310 & 3.84492 & 1.52299 & 0.51563 & 3.76836 \\
\hline & 0.9 & 2.00627 & 0.48247 & 3.93986 & 1.91454 & 0.48304 & 3.92098 & 1.53822 & 0.48556 & 3.84111 \\
\hline
\end{tabular}

Table 1 indicates results for wall values for the gradients of velocity, temperature and volume fraction functions which are proportional to the friction factor, Nusselt number and Sherwood number, respectively. From this table, we notice that as $N_{r}$ increases, the friction factor increases, the heat transfer rate (Nusselt number) and mass transfer rate (Sherwood number) decrease. As $N_{t}$ and $N_{b}$ increase, the friction factor and surface mass transfer rates increase whereas the surface heat transfer rate decreases

Table (1). Effect of $N t, N b$ and $N r$ on $f^{\prime \prime}(0), \theta^{\prime}(0)$ and $\phi^{\prime}(0)$ with $A=0.5, \lambda=1, \gamma=0.5, \Delta=0.5, \operatorname{Pr}=10$ and Le $=10$ 


\begin{tabular}{ccccccccccc}
\hline & 0.1 & 1.96866 & 0.48162 & 3.52373 & 1.87681 & 0.48224 & 3.51037 & 1.50008 & 0.48497 & 3.45396 \\
& 0.3 & 1.98501 & 0.44299 & 3.63003 & 1.89347 & 0.44363 & 3.61569 & 1.51811 & 0.44640 & 3.55514 \\
0.7 & 0.5 & 1.99883 & 0.41555 & 3.71507 & 1.90787 & 0.41617 & 3.70001 & 1.53507 & 0.41889 & 3.63650 \\
& 0.7 & 2.01059 & 0.39448 & 3.78574 & 1.92032 & 0.39508 & 3.77015 & 1.55044 & 0.39771 & 3.70439 \\
& 0.9 & 2.02073 & 0.37744 & 3.84655 & 1.93115 & 0.37802 & 3.83051 & 1.56425 & 0.38055 & 3.76297 \\
\hline
\end{tabular}

The effects of the first and second resistances of the porous medium on the gradients of velocity, temperature and volume fraction functions are been illustrated in Table 2. From this table we conclude that both the first and second resistances enhance the wall shear stress and the mass transfer rate and reduce the heat transfer rate.

Table (2). Effects of $\gamma$ and $\Delta$ on $f^{\prime \prime}(0),-\theta(0)$ and $-\phi^{\prime}(0)$ with $A=0.5, \lambda=1$, $N_{T}=0.5, N_{B}=0.5, N_{R}=1, \operatorname{Pr}=10$ and $L e=10$

\begin{tabular}{lllll}
\hline $\boldsymbol{\gamma}$ & $\boldsymbol{\Delta}$ & $\mathbf{f}^{\prime \prime}(\mathbf{0})$ & $\boldsymbol{- \theta}(\mathbf{0})$ & $\boldsymbol{- \phi ( 0 )}$ \\
\hline \multirow{4}{*}{0.0} & 0.0 & 1.74114 & 0.55465 & 3.71225 \\
& 2.0 & 2.42814 & 0.54777 & 3.86815 \\
& 4.0 & 2.94365 & 0.54481 & 3.96362 \\
& 6.0 & 3.37615 & 0.54328 & 4.03332 \\
2.0 & 0.0 & 2.27894 & 0.54976 & 3.82839 \\
& 2.0 & 2.82588 & 0.54591 & 3.93648 \\
& 4.0 & 3.27555 & 0.54397 & 4.01254 \\
4.0 & 6.0 & 3.66738 & 0.54292 & 4.07146 \\
& 0.0 & 2.69411 & 0.54729 & 3.90496 \\
& 2.0 & 3.16479 & 0.54480 & 3.98909 \\
& 4.0 & 3.56984 & 0.54347 & 4.05272 \\
6.0 & 6.0 & 3.93132 & 0.54275 & 4.10394 \\
& 0.0 & 3.05265 & 0.54579 & 3.96396 \\
& 2.0 & 3.47213 & 0.54411 & 4.03302 \\
& 4.0 & 3.84350 & 0.54318 & 4.08770 \\
& 6.0 & 4.18059 & 0.54269 & 4.13297 \\
\hline
\end{tabular}

We computed the solutions for the dimensionless velocity, temperature and nanoparticle volume fraction in Figs. 2-10. The effects of all the parameters governing the problem are discussed.

The variation of the non-dimensional velocity, temperature and nanoparticle concentration for $N_{t}=0.5, N_{b}=0.5, N_{r}=1.0, \lambda=1.0, \operatorname{Pr}=10, L e=10, \gamma=0.5, \Delta=$ with unsteadiness parameter $A$ is illustrated in Fig. 2. It can be observed from Fig. 2(a) that first for velocity distribution, there is a special point ( $\eta \approx 1.4$ ) called 'crossing over point' and the velocity profiles have completely conflicting behavior before and after that point. The value of the velocity profile for fixed $\eta$ increases before that point and slightly decreases after that. Thus, due to the increase of unsteadiness parameter, $A$, the velocity initially enhances but ultimately it increases the thickness of momentum boundary layer. On the other hand the temperature and nanoparticle volume fraction profiles of Figs. 2 (b, c), show that the unsteadiness controls the heat and mass transfer. The control of heat and mass transfer is of great practical significance. For the increase of $A$, the temperature and nanoparticle volume fraction at a point decreases because the thermal boundarylayer thickness rapidly decreases due to increase of unsteadiness.

The effect of mixed convection parameter $\lambda$ on the nondimensional velocity, temperature and nanoparticle volume fraction is illustrated in Fig. 3. From these figures, it is observed that the velocity is increased as $\lambda$ increase, but both the temperature and nanoparticle fraction decrease with increasing $\lambda$.

The velocity profile decreases and both the temperature and nanoparticle volume fraction increases as nanofluid buoyancy ratio parameter $N_{r}$ increases as showed in in Fig. 4.

Figure 5 presents the effect of thermophoresis $N_{t}$ on the velocity, temperature and volume fraction distributions. It is observed that the momentum boundary-layer thickness increases with an increase of $N_{t}$. As the parameter $N_{t}$ increases, the thermal and nanoparticle volume fraction boundary-layer thickness increase for the specified conditions.

Furthermore, increasing the value of the Brownian motion $N_{b}$ causes a thickening of momentum and thermal boundary layers, whereas thinning of the nanoparticle volume fraction boundary layer as shown in Fig.6. Physically, it is true due to the fact that the large values of the Brownian motion parameter impacts a large extent of the fluid. It results in the thickening of the momentum and thermal boundary layers. Hence, the present analysis shows that the flow field is appreciably influenced by the Brownian motion $N_{b}$.

The effect of both first resistant parameter $\gamma$ and second resistant parameter $\Delta$ is showed in Fig. 7 and Fig. 8, respectively. From these figures, it is observed that both $\gamma$ and $\Delta$ have the same behavior. The velocity profile increases but the temperature profile and volume fraction profile decreases as both $\gamma$ and $\Delta$ increases.
From these figures, it is observed that as Pr increases the velocity and temperature profiles decrease. On the other hand, for the volume fraction profiles there is a crossing over point at ( $\eta \approx 0.5$ ) where the volume fraction profile decreases before that point and slightly increases after that.

It is noticed, from Fig. 10 that an increase in the Lewis number $L e$ results in an increase in the velocity, but in a decrease of the volume fraction within the boundary layer. In the temperature profile there is a crossing over point at ( $\eta \approx 0.8$ ) where the temperature profiles have completely conflicting behavior before and after that point. The value of the temperature profile for fixed $\eta$ decreases before that point and slightly increases after it. The present analysis shows that the flow field is appreciably influenced by the Lewis number Le. 
(a)

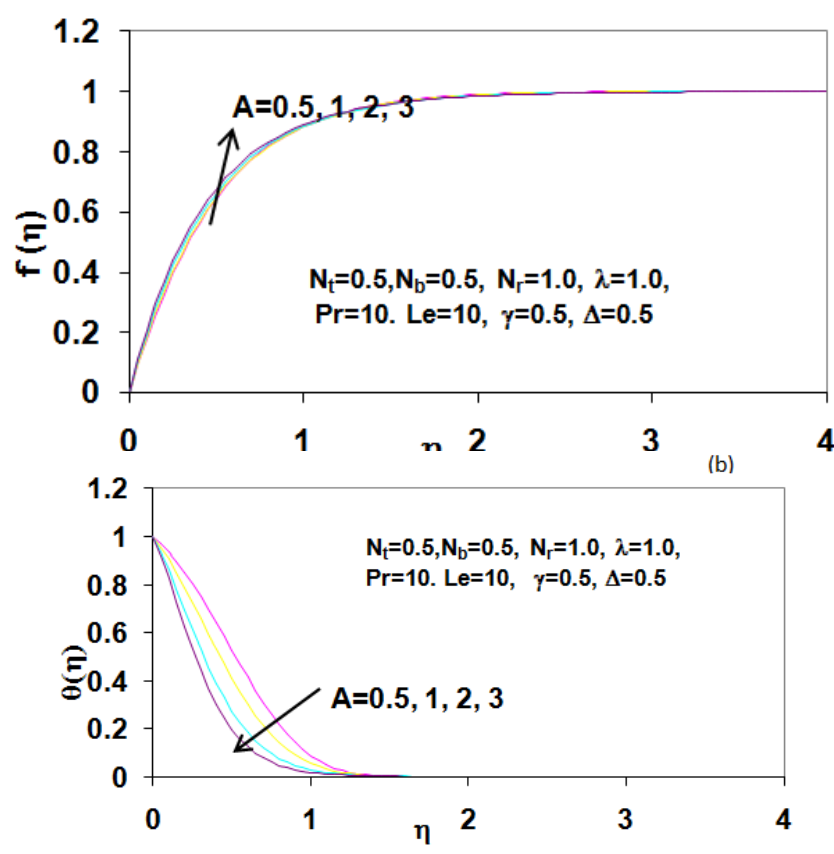

(c)

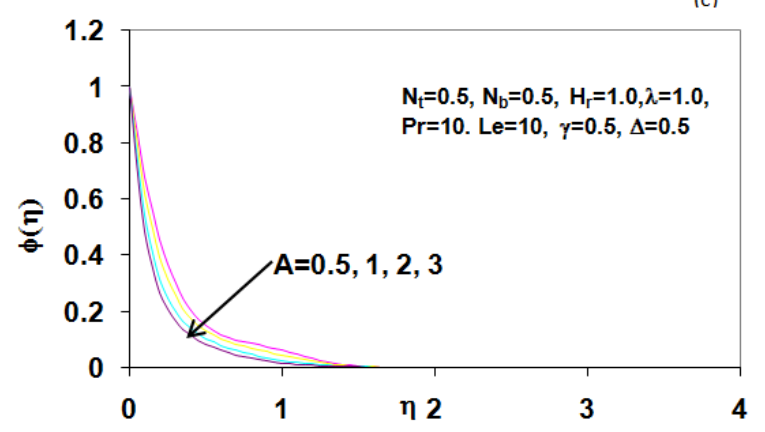

Fig. 2. Effects of unsteadiness parameter $A$ on (a) velocity, (b) Temperature and (c) Nanoparticle volume fraction profiles.

(a)

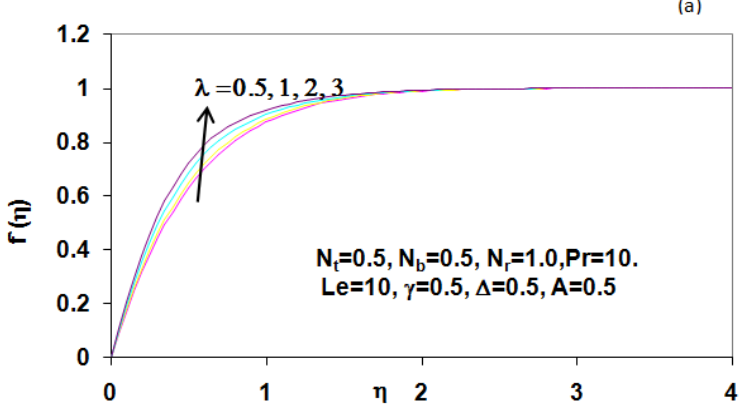

(b)

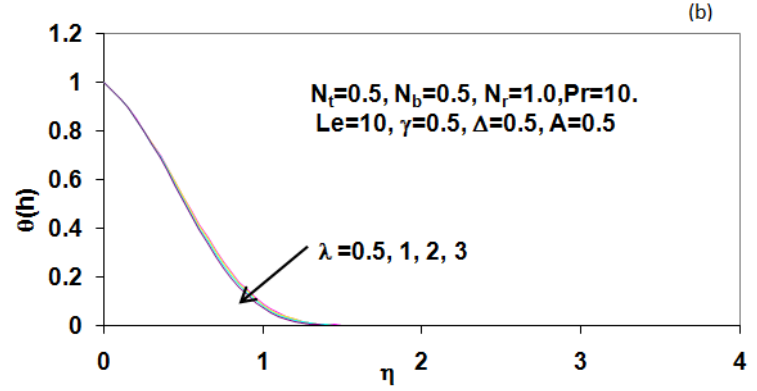

(c)

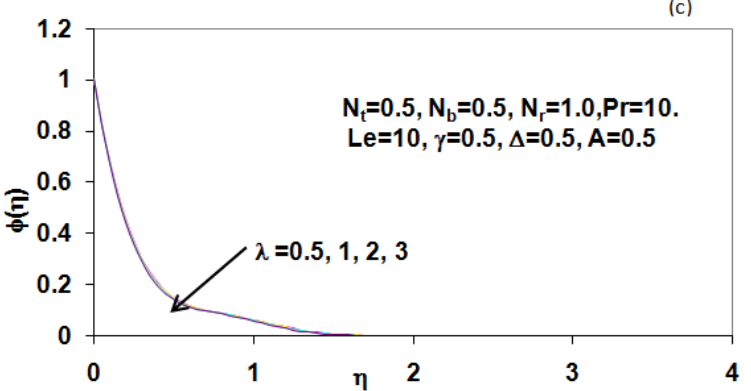

Fig. 3. Effects of mixed convection parameter $\lambda$ on (a) velocity, (b) Temperature and (c) Nanoparticle volume fraction profiles.

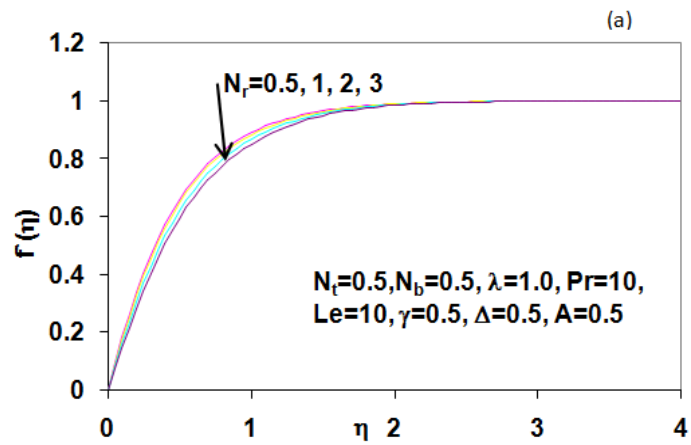

(b)

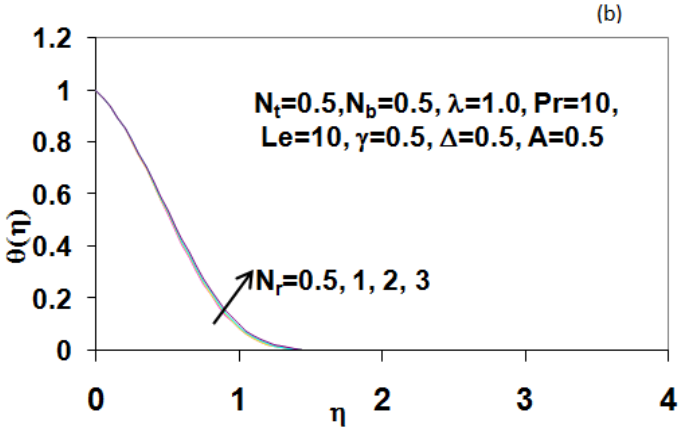

(c)

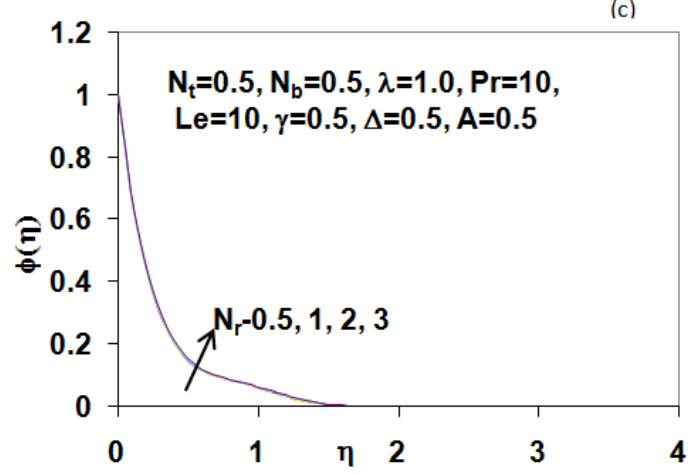

Fig. 4 Effects of nanofluid buoyancy ratio parameter $N_{r}$ on (a) velocity, (b) Temperature and (c) Nanoparticle volume fraction profiles. 
48 A. A. Abdullah, F. S. Ibrahim et al.: Investigation of Unsteady Mixed Convection Flow near the Stagnation Point of a Heated Vertical Plate embedded in a Nanofluid-Saturated Porous Medium by Self-Similar Technique

(a)

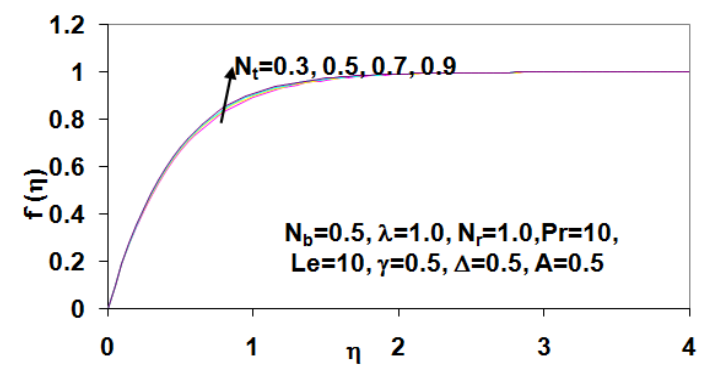

(b)
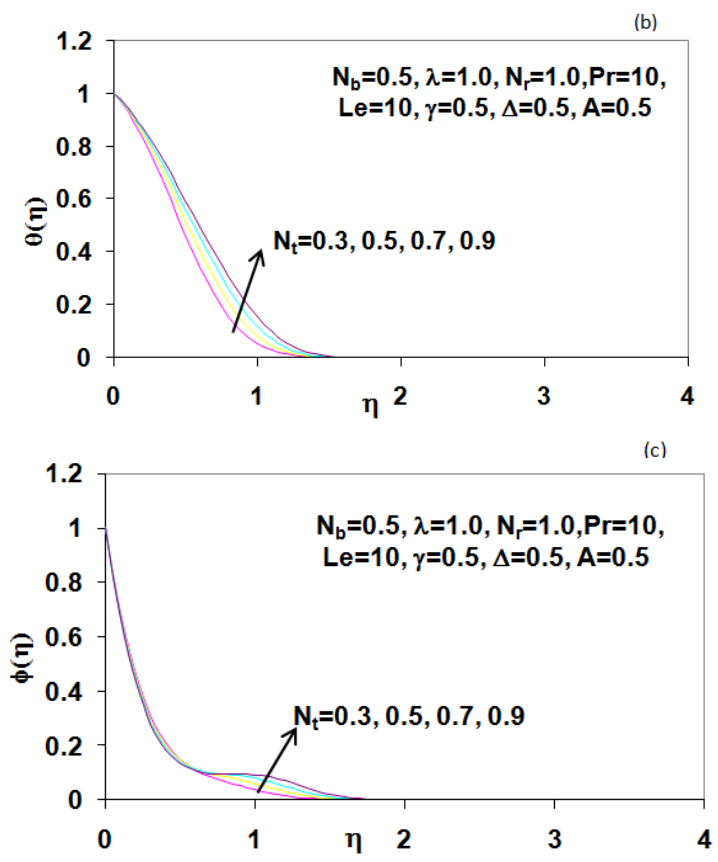

Fig. 5. Effects of thermophoresis parameter $N_{t}$ on (a) velocity, (b) Temperature and (c) Nanoparticle volume fraction profiles.

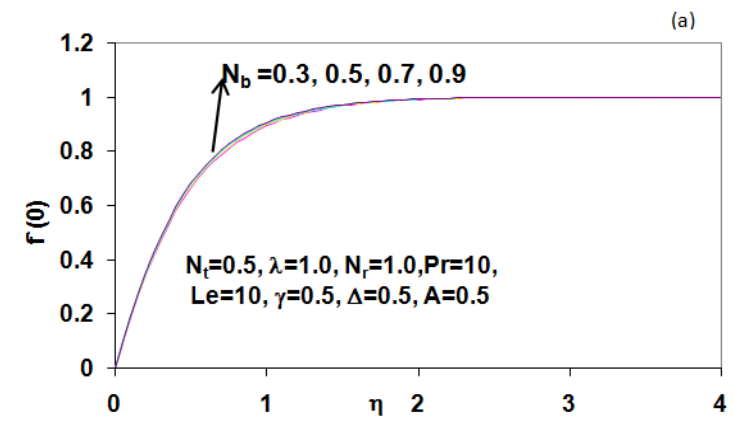

(b)

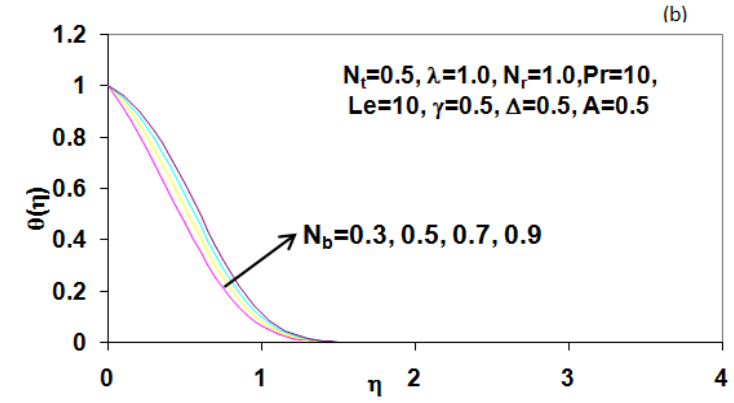

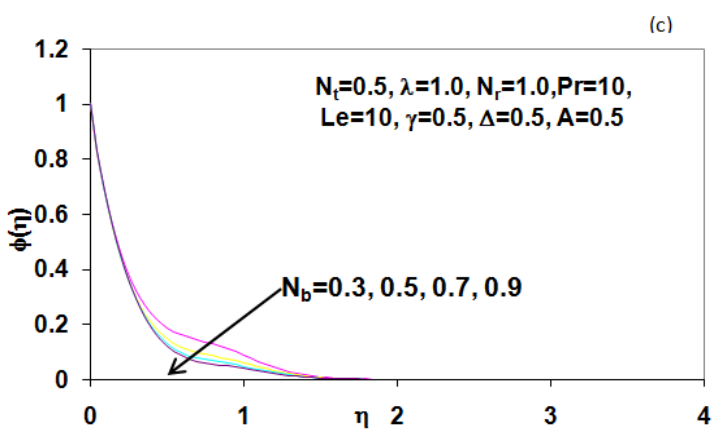

Fig. 6. Effects of Brownian motion parameter $N_{b}$ on (a) velocity, (b) Temperature and (c) Nanoparticle volume fraction profiles.
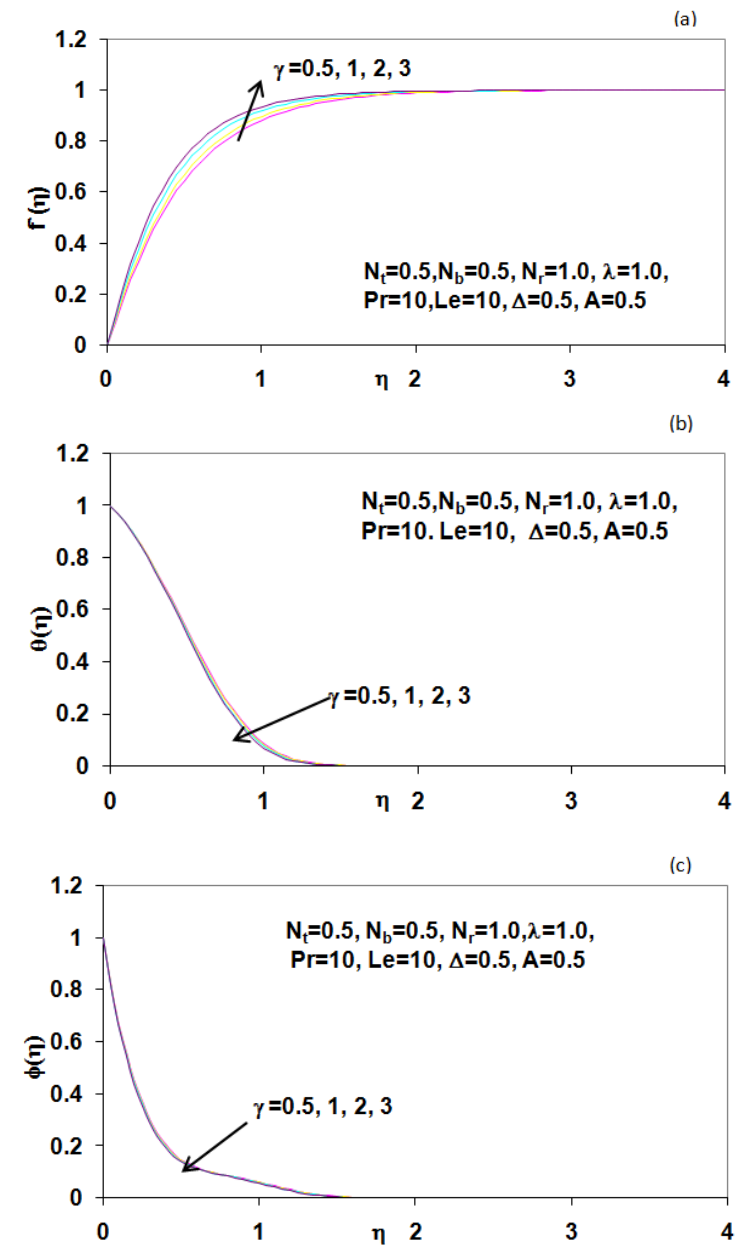

Fig. 7. Effects of first resistant parameter $\gamma$ on (a) velocity, (b) Temperature and (c) Nanoparticle volume fraction profiles.

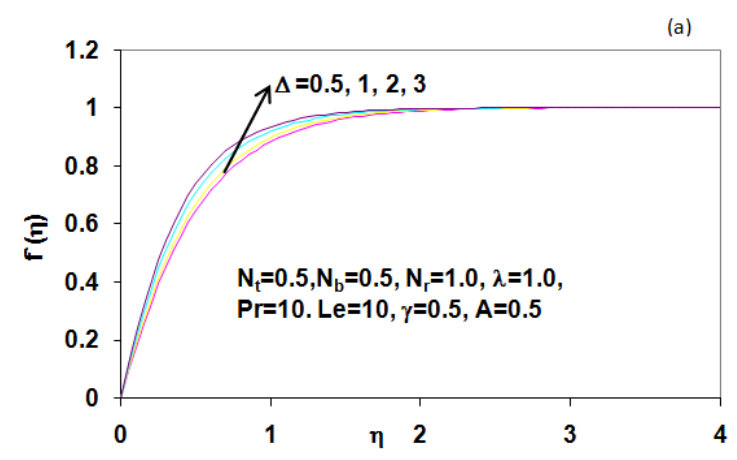


(b)

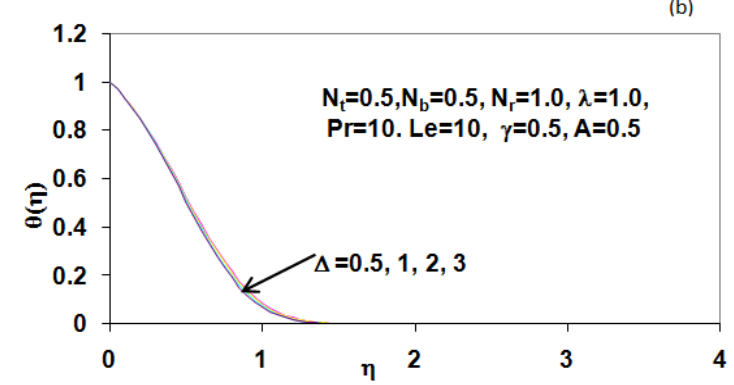

(c)

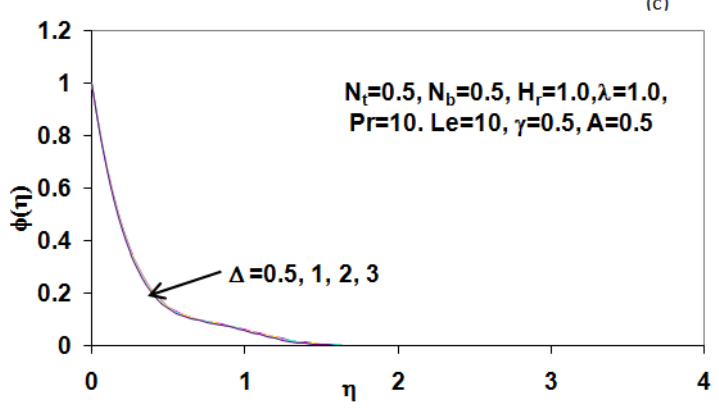

Fig. 8. Effects of second resistant parameter $\Delta$ on (a) velocity, (b) Temperature and (c) Nanoparticle volume fraction profiles.

(a)

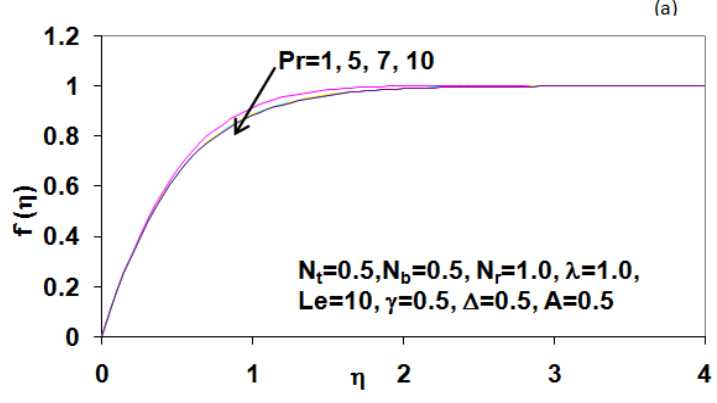

(b)

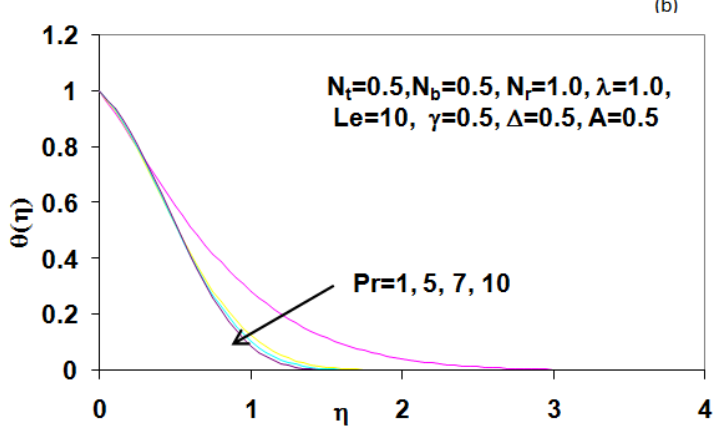

(c)

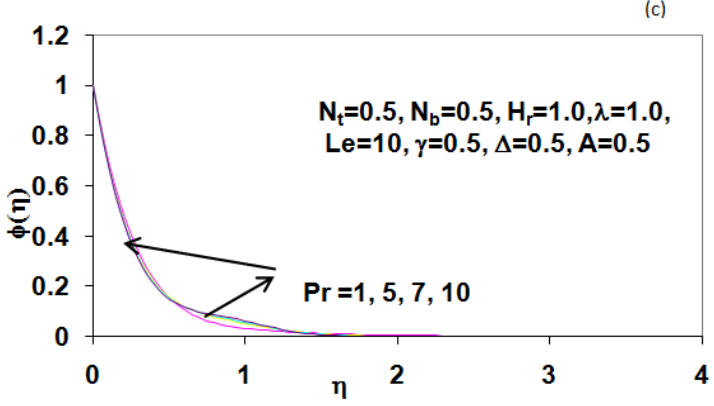

Fig. 9. Effects of Prandt number $\operatorname{Pr}$ on (a) velocity, (b) Temperature and (c) Nanoparticle volume fraction profiles. (a)

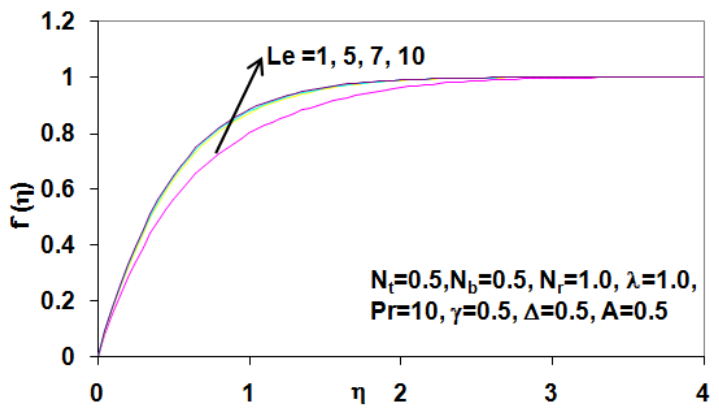

(b)

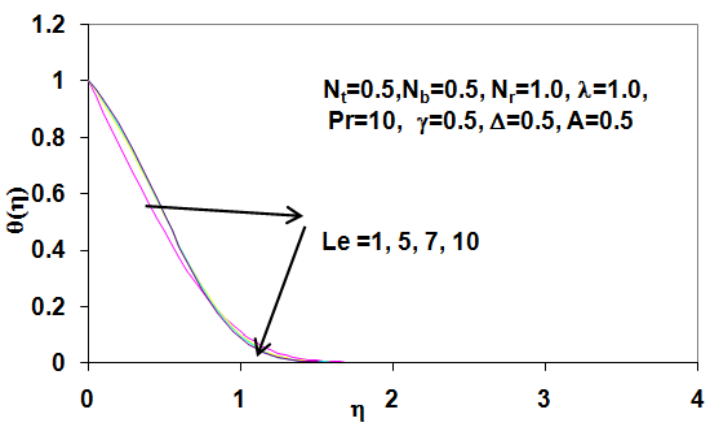

(c)

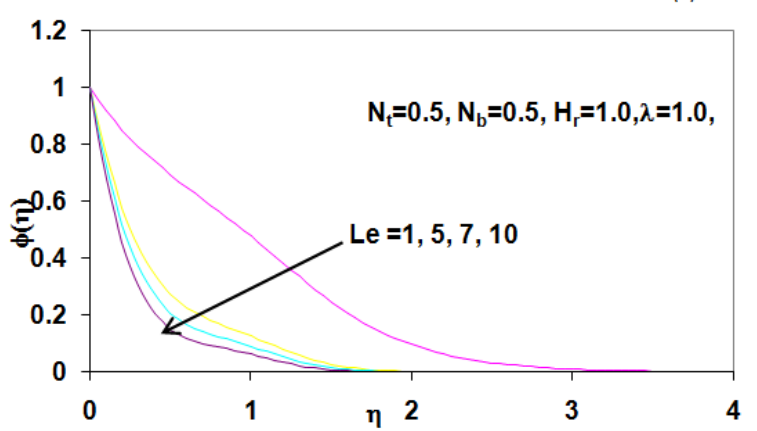

Fig. 10. Effects of Lewis number Le on (a) velocity, (b) Temperature and (c) Nanoparticle volume fraction profiles.

\section{Conclusions}

In the present work, we studied theoretically the problem of unsteady mixed convection boundary-layer flow near a stagnation point of a heated vertical surface embedded in a nanofluid-saturated porous medium. The resulting system of nonlinear partial differential equations is transformed to a system of ordinary differential equations by the means of self-similar solution. The obtained system is solved numerically using an efficient numerical shooting technique with a fourth-fifth order Runge-Kutta method scheme (MATLAB package). The solutions for the flow, heat and mass transfer characteristics are evaluated numerically for various values of the governing parameters, namely unsteadiness parameter $A$, mixed convection parameter $\lambda$, nanofluid buoyancy ratio parameter $N_{r}$, thermophoresis parameter $N_{t}$, Brownian motion parameter $N_{b}$, first resistant parameter $\gamma$, second resistant parameter $\Delta$. Prandtl number Pr and Lewis number Le .

The following are brief summary conclusions drawn from 
the analysis:

1. The magnitude of the skin friction coefficient $f^{\prime \prime}(0)$ increases, while the local Nusselt number decreases with increasing the nanofluid parameters $N_{t}, N_{b}$ and $N_{r}$.

2. The local Sherwood number increases as both $N_{t}$ and $N_{b}$ parameter increases. However, it decreases as $N_{r}$ increases.

3. The thickness of momentum boundary layer decreases with an increase in nanofluid buoyancy ratio parameter $N_{r}$. and Prandtl number $\operatorname{Pr}$. However, it increases with increasing all other parameters.

4. The thickness of thermal boundary layer increases with an increase in the nanofluid parameters $N_{t}, N_{b}$ and $N_{r}$.

5. The nanoparticle volume fraction boundary-layer thickness increases with an increase in both $N_{t}$ and $N_{r}$ parameters and decreases with an increase in $N_{b}$ parameter.

6. Both the first and second resistance parameters $\gamma$ and $\Delta$ enhanced the momentum boundary-layer thickness and reduced both the thermal and nanoparticle volume fraction boundary-layer thickness

\section{Acknowledgement}

The authors would like to thank National Science, Technology and Innovation Plan (NSTIP) at Kingdom of Saudi Arabia (Project ID 12-MAT2296-10) for the financial support.

\section{Nomenclature}

$\begin{array}{ll}A & \text { Unsteadiness parameter } \\ a, b, c & \text { Constants in Eq. (6) } \\ C_{f} & \text { Skin friction coefficient } \\ \mathrm{D}_{\mathrm{a}} & \text { Darcy number } \\ \mathrm{D}_{\mathrm{B}} & \text { Brownian diffusion coefficient } \\ \mathrm{D}_{\mathrm{T}} & \text { Thermophoresis diffusion coefficient } \\ f & \text { dimensionless stream function } \\ \mathrm{G} & \text { Acceleration due to gravity } \\ \mathrm{Gr} & \text { Grashof number } \\ \mathrm{Le} & \text { Lewis number } \\ \mathrm{k} & \text { Thermal conductivity } \\ K & \text { Permeability } \\ \mathrm{N}_{\mathrm{b}} & \text { Brownian motion parameter } \\ \mathrm{N}_{\mathrm{r}} & \text { Nanofluid buoyancy ratio parameter } \\ \mathrm{N}_{\mathrm{t}} & \text { Thermophoresis parameter } \\ \mathrm{Pr} & \text { Prandtl number } \\ \mathrm{Re} & \text { Local Reynolds number } \\ \mathrm{Sh} & \text { Sherwood number } \\ t & \text { Time } \\ \mathrm{T} & \text { Fluid temperature } \\ \mathrm{T}_{\mathrm{w}} & \text { Temperature at the surface } \\ \mathrm{T}_{\infty} & \text { Ambient temperature as y tends to } \\ & \text { infinity }\end{array}$

$\begin{array}{ll}u, v & \begin{array}{l}\text { Velocity components along } x \text { and } y \\ \text { directions, respectively }\end{array} \\ x, y & \begin{array}{l}\text { Distances along and normal to the } \\ \text { surface }\end{array} \\ \begin{array}{ll}\text { Greek symbols } & \text { Thermal diffusivity } \\ \alpha & \text { Coefficient of volumetric thermal } \\ \beta & \text { expansion } \\ \varphi & \begin{array}{l}\text { Dimensionless nanoparticle volume } \\ \text { fraction }\end{array} \\ \Gamma & \text { Empirical constant } \\ \theta & \text { Pseudo similarity variable } \\ \lambda & \text { Dimensionless temperature } \\ \mu & \text { Buoyancy parameter } \\ \gamma & \text { Coefficient of viscosity } \\ \frac{\partial u}{\partial x}+\frac{\partial v}{\partial y}=0 & \text { First order resistance } \\ v & \text { Second order resistance } \\ \text { Subscripts } & \text { Kinematic viscosity } \\ e, w, \infty & \text { Conditions at the edge of the } \\ & \text { boundary layer, at the surface and in } \\ \text { the free stream }\end{array}\end{array}$

\section{References}

[1] Ramachandra N., Chen T. and Armaly B, 1988. Mixed convection in the stagnation flows adjacent to vertical surface, J. Heat Transfer Vol. 110, pp. 173-177.

[2] Scshadri R., Srccshylan N. and Nath G., 2002. Unsteady mixed convection flow in the stagnation region of a heated vertical plate due to impulsive motion, Int. J. Heat and Mass Transfer Vol. 45 pp. 1345-1352.

[3] Hall M., 1969. The boundary layer over an impulsively started flat plate, Proc. R. Soc. Vol. 310A, pp. 401-414.

[4] Dennis S., 1972. The motion of a viscous fluid past an impulsively started semi-infinite flat plate, J. Inst. Math. It's Appl. Vol. 10, pp. 105-117.

[5] Watkins C., 1975. Heat transfer in the boundary layer over an impulsively started flat plate, J. Heat Transfer Vol. 97, pp. 492-484.

[6] Smith S., 1967. The impulsive motion of a wedge in a viscous fluid, Z. Angew. Math. Phys. Vol. 18 , pp. 508-522.

[7] Nanbu K., 1971. Unsteady Falkner Skan flow, Z. Angew. Math. Phys. Vol. 22, pp. 1167-1172.

[8] Williams J. and Rhyne T.,1980. Boundary layer development on a wedge impulsively set into motion, SIAM J. Appl. Math. Vol. 38, pp. 215-224.

[9] Kumari M., 1997. Development of flow and heat transfer on a wedge with a magnetic field, Arch. Mech. Vol. 49, pp. 977-990.

[10] Ece M., 1992. An initial boundary layer flow past a translating and spinning rotational symmetric body, J. Eng. Math. Vol. 26, pp. 415-428. 
[11] Ozturk A. and Ece M., 1995. Unsteady forced convection heat transfer from a translating and spinning body, J. Energy Rcsour. Tcchnol. Vol. 117, pp. 318-323.

[12] Brown S. and Riley N., 1973. Flow past a suddenly heated vertical plate, J. Fluid Mech. Vol. 59, pp. 225-237.

[13] Ingham D., 1985. Flow past a suddenly heated vertical plate, Proc. R. soc. Vol. 402A, pp. 109-134.

[14] Amin N. and Riley N., 1995. Mixed convection at a stagnation point, Quart. J. Mech. Vol. 48, pp. 111-121.

[15] Hassanien I., Ibrahim, F. and Omer Gh.,2004. Unsteady free convection flow in the stagnation-point region of a rotating sphere embedded in a porous medium, Mech.Mech. Eng. Vol. 7, pp. 89-98.

[16] Hassanien, I., Ibrahim, F. and Omer Gh.,2006. Unsteady flow and heat transfer of a viscous fluid in the stagnation region of a three-dimensional body embedded in a porous medium, J.Porous Media, Vol. 9, pp. 357-372.

[17] Hassanien, I. and Al-Arabi, T.,2008. Thermal Radiation and variable viscosity effects on unsteady mixed convection flow in the stagnation region on a vertical surface embedded in a porous medium with surface heat flux Vol. 29, pp. 187 - 207.

[18] Yacob N., Ishak A. and Pop I., 2011. Falkner-Skan problem for a static or moving wedge in nanofluids. Int J Thermal Sci. Vol 50, pp. 133-139.

[19] Congedo P., Collura S. and Congedo P., 2009.Modeling and analysis of natural convection heat transfer in nanofluids. In: Proc ASME Summer Heat TransferConf. Vol. 3,pp. 569-579.

[20] Hamad M., Pop I. and Ismail A. 2011. Magnetic field effects on free convection flow of a nanofluid past a semi-infinite vertical flat plate. Nonlinear Analysis:Real World Appl. Vol. 12, pp. 1338-1346.
[21] Buongiorno J., 2006. Convective transport in nanofluids. ASME J Heat Transfer.Vol. 128, pp. 240-250.

[22] Hamad M., 2011. Analytical solution of natural convection flow of a nanofluid over a linearly stretching sheet in the presence of magnetic field. Int. Commun. Heat Mass Transfer. Vol. 38, pp. 487-492.

[23] Khan W and Pop I.,2010. Boundary-layer flow of a nanofluid past a stretching sheet. Int. J Heat Mass Transfer. Vol. 53, pp. $2477-2483$.

[24] Abu-Nada E. and Chamkha A., 2010. Effect of nanofluid variable properties on natural convection in enclosures filled with a $\mathrm{CuO}-\mathrm{EG}-$ water nanofluid. Int. J. Thermal Sci. Vol. 49, pp. 2339-2352.

[25] Das S., S Choi SU, Yu W. and Pradeep T., 2007. Nanofluids: Science and Technology. New Jersey: Wiley.

[26] Kakaç S. and Pramuanjaroenkij A., 2009. Review of convective heat transfer enhancement with nanofluids. Int. J Heat Mass Transfer. Vol. 52, pp. 3187-3196.

[27] Muthtamilselvan M., Kandaswamy P. and Lee J., 2010. Heat transfer enhancement of copper-water nanofluids in a liddriven enclosure. Commun Nonlinear Sci. Numer. Simulat. Vol. 15, pp. 1501-1510.

[28] Pereyra V.,1978. PASVA3, an adaptive finite difference FORTRAN program for first order non-linear boundary value problems, in: Lecture Note in Computer Science, Vol. 76, Springer, Berlin.

[29] Scshadri R., Srccshylan N. and Nath G., 2002. Unsteady mixed convection flow in the stagnation region of a heated vertical plate due to impulsive motion, Int. J. Heat and Mass Transfer Vol. 45 pp. 1345-1352. 